\title{
(Demo) Physical Visualization Design
}

\author{
Lana Ramjit \\ lana@cs.ucla.edu \\ UCLA \\ Ravi Netravali \\ ravi@cs.ucla.edu \\ UCLA
}

\begin{abstract}
We demonstrate PVD, a system that visualization designers can use to co-design the interface and system architecture of scalable and expressive visualization.

\section{ACM Reference Format:}

Lana Ramjit, Zhaoning Kong, Ravi Netravali, and Eugene Wu. 2020. (Demo) Physical Visualization Design. In Proceedings of the 2020 ACM SIGMOD International Conference on Management of Data (SIGMOD'20), June 14-19, 2020, Portland, OR, USA. ACM, New York, NY, USA, 4 pages. https://doi.org/10.1145/3318464.3384711
\end{abstract}

\section{INTRODUCTION}

Building interactive data visualizations is hard. It requires expertise spanning human-computer interaction, networking, and database optimization. Visualization designers need to ensure that the interface's visual layout is expressive enough to accomplish the desired user tasks. At the same time, designers also need to make architectural and systems optimization decisions in order to ensure that the interface is responsive in the face of large and growing data sizes.

The processes of designing an effective interface and developing a responsive architecture are intertwined: the interface and interaction design determine the data flows expressible by the user, while the architecture design determines the scale at which these data flows can execute quickly enough. For instance, an interface consisting of a single small dropdown menu can ensure interactive speeds by pre-computing and caching the query results associated with each of the options. However, this strategy fails when adding an interaction

Permission to make digital or hard copies of all or part of this work for personal or classroom use is granted without fee provided that copies are not made or distributed for profit or commercial advantage and that copies bear this notice and the full citation on the first page. Copyrights for components of this work owned by others than the author(s) must be honored. Abstracting with credit is permitted. To copy otherwise, or republish, to post on servers or to redistribute to lists, requires prior specific permission and/or a fee. Request permissions from permissions@acm.org. SIGMOD'20, June 14-19, 2020, Portland, OR, USA

(C) 2020 Copyright held by the owner/author(s). Publication rights licensed to the Association for Computing Machinery.

ACM ISBN 978-1-4503-6735-6/20/06 . .\$15.00

https://doi.org/10.1145/3318464.3384711

\author{
Zhaoning Kong \\ jonnykong@cs.ucla.edu \\ UCLA \\ Eugene $\mathrm{Wu}$ \\ ewu@cs.columbia.edu \\ Columbia University
}

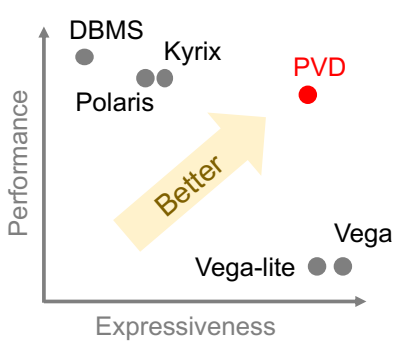

Figure 1: Current visualization frameworks trade-off expressiveness and performance.

such as free-text search, which would require an inordinate amount of pre-computation storage, and thus necessitates a different architectural design strategy.

The complexity of such design decisions poses a major practical challenge because creating new visualization interfaces is not a one-shot process. Instead, designers iteratively create prototypes, using the feedback from their intended users to refine the design, add new views and interactions, and ensure that the interactions are sufficiently responsive. If the data size is negligible, then the designer can focus solely on interface design, which is well-supported by existing visualization [9] and design [2, 6] tools. However, if the datasets are large, then simply creating a prototype requires setting up a server that connects to a data management system, and making physical database design and caching optimizations so the prototype is responsive. Even if the designer is capable of this engineering work, the tremendous engineering cost can "lock-in" the designer to early architecture decisions.

There is a need for tools that support the rapid codesign of the visualization interface and the system architecture. Unfortunately, existing tools primarily focus on one of the two aspects (Figure 1). At the extremes, visualization libraries like Vega-lite [9] help accelerate client-side visualization design, whereas database tuners [1,3] optimize the physical data layouts but are agnostic to the application interface design. Scalable visualization frameworks like Kyrix [10], Falcon [8], and Polaris [7] make specific architectural decisions that limit the designer to a subset of designs or interactions that the architecture can efficiently express, e.g., pan/zoom or brushed linking. 
To overcome these limitations, PVD is a co-design tool that helps visualization designers rapidly iterate through the combinatorial space of interface designs and physical layouts. The key challenge is in identifying the appropriate abstraction for specifying PVD's inputs. It must be low-level enough to express a wide range of visualization interfaces, yet high-level enough to enable effective optimization. To address this tension, PVD models the interface as a set of data flows (SQL queries) that are structurally transformed and executed in response to user interactions.

Thus, PVD takes as input a specification of the interface design, architectural optimization techniques, constraints on the available resources, and expectations of interactivity and responsiveness. For instance, the designer may specify that the dataset can vary from $2 \mathrm{MB}$ to $2 \mathrm{~GB}$, server and client memory are $2 \mathrm{~GB}$ and $150 \mathrm{MB}$ respectively, and that she expects interactions to be serviced within 50ms. PVD then outputs the expected response times of the interactions in the interface and, if the constraints cannot be met, recommends ways to modify the interface or architectural designs. The estimated response times are directly simulated in the design interface, so the designer can directly experience the effects of different architecture choices across the system.

In this demonstration, users will use PVD to interactively build a scalable visualization interface by adding visual components and interactions, and seeing how small interface changes affect the utility of different architectural designs. At each step, users will receive immediate feedback on the visualization's performance and design. Once a user is satisfied, PVD will instantiate and deploy the designed interface.

\section{USE CASE}

iCheckuClaim [11] is a web-based visualization application developed by database researchers at Duke University. Users explore and contextualize U.S. politician voting records as compared to peer groups (e.g., Republican/Democratic majority, the President, etc). We describe the interface design and architecture of a subset of three visualizations (called "views") in the main interface (Figure 2).

\subsection{Interface Design}

(V1) Ranked Politicians Histogram: The sorted bar chart lists politicians within a demographic group (e.g., senators, female house reps, etc) along the $\mathrm{x}$-axis. The $\mathrm{y}$-axis shows the percentage of votes cast by a politician that aligned with the position of a user-selected peer group. Users pick each of the two groups from a pre-defined list. The orange bar is the currently selected politician (e.g., Senator Feinstein).

(V2) Vote Count Histogram: The total number of votes per week over five years. Users select a date range (shaded

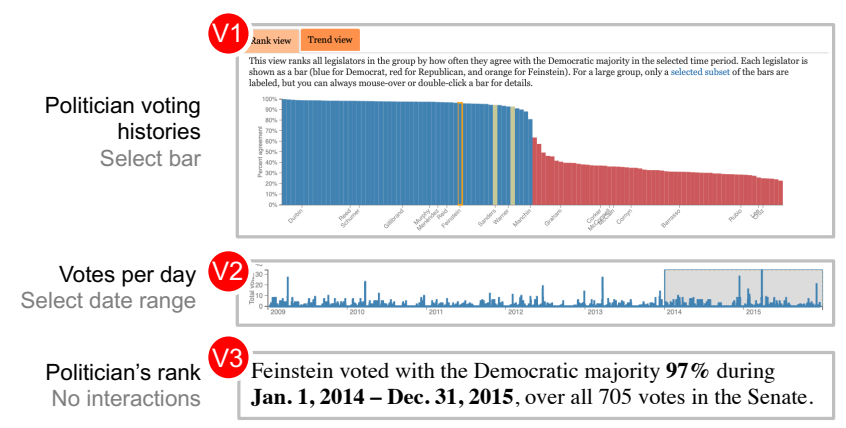

Figure 2: Three example iCheckuClaim charts (views).

region), which will update the percentages in V1 based on votes in the selected range, and update V3 (described next).

(V3) Politician Text Summary: Describes a specific politician's rank and percentage of agreement within the currently selected time window. This is updated when a new bar (representing a new politician) is selected from $\mathrm{V} 1$ and when the selection in V2 changes.

\subsection{System Architecture}

iCheckuClaim uses a client-server architecture. The backend data store is a ReDis instance which stores the raw voting data. Additionally, prefix-sum indexes for common peer groups (such as the President) are pre-computed and cached in-memory. Other peer groups compute a prefix-sum index on the fly. Requested indexes are sent to the client.

The Javascript client caches all data received from the server, and reloads the page when a new peer group is selected. As users interact with the interface, event handlers decide whether to update the interface using the client cache or send a server request. The cached prefix-sum index can recalculate a politician's voting behavior in constant time as the user selects new date ranges or different politicians.

\subsection{Challenges}

Even in this simple interface, subtle interface design decisions have considerable affect on the architecture. For instance, pre-defining the peer groups limits the user's choices but enables pre-computation. Rendering V1, V2, and V3 on the same page implies that the user will expect V1 to quickly update as the user creates and resizes a selection in V2. Further, the designer must choose whether the selection should continuously update V1 and V3 as it is manipulated, or only when the user finishes the selection interaction.

Each of these choices adds or removes architectural requirements in terms of the data structures, caching, and data placement choices that must be made to meet the user expectations. However, choosing to materialize an index to accelerate the date range interaction can reduce the resources available for pre-computing and optimizing interactions in 


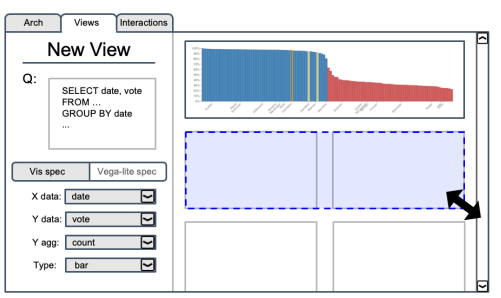

(a) Add view

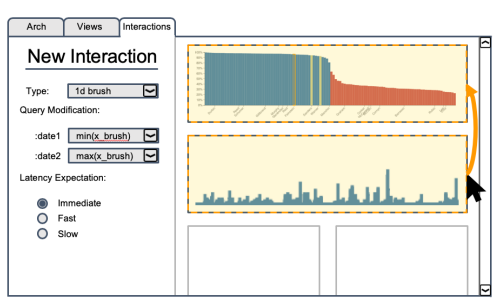

(b) Add interaction

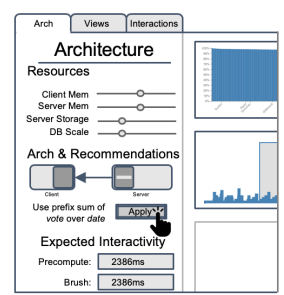

(c) Apply opt.

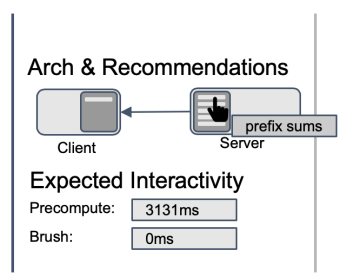

(d) Updated latency profile

Figure 4: Screenshot mockups of the demo walkthrough.

Our current approach is inspired by physical database design solutions [4]. We enumerate each combination of sampled queries, relevant optimizations, and data placements, modeling each combination as an integer. PVD solves the resulting integer linear programming problem, whose solution is a recommended architecture and latency profile. Although PVD currently relies on naive workload sampling to prevent combinatorial explosion problem, we plan to explore and implement query compression techniques that will enable PVD to directly optimize the visualization specification.

\section{DEMONSTRATION}

Users will build an interface akin to iCheckuClaim using the same voting dataset, and experience the interface-architectural design trade-offs first hand. Below, we describe an example walk-through of the demo experience (Figure 4).

(a) The interface lays views in a grid (light gray baxes). The user adds a new view by selecting a rectangle of the desired size. This shows the New View tab on the left, where the user specifies the query and visualization spec. In this example, the user adds the votes by date view.

(b) The user creates an interaction by dragging an edge from the source to destination view. The New Interaction tab lets the user specify the interaction in the source view (e.g., $1 \mathrm{~d}$ brush) and how it transforms the query in the target view (filter the date predicate). Users can optionally specify their expectations for the responsiveness of this interaction.

(c) The architecture tab displays the current architecture, and has sliders to specify resource constraints (client, server, network) and to re-scale the database. PVD solves the optimization problem to find that the existing architecture, which does not materialize any data structures, will have poor responsiveness. It recommends adding a prefix-sum data structure to accelerate the brushing interaction, which the user can then choose to Apply.

(d) Accepting the prefix-sum optimization immediately updates the architecture diagram and latency profile. This optimization caches a file on the client on page load, increasing the download time slightly from $2387 \mathrm{~ms}$ to $3131 \mathrm{~ms}$. However, all ensuing interactions are sub-millisecond in-memory lookups thanks to the prefix-sum index on the client. This is reflected in the updated latency profile estimates.
Finally, PVD will instantiate the interface into a full-fledged web application, apply the optimizations, and manage the client-server communication and caching. The query results are also exposed as tables in the client Javascript code, so the designer can benefit from the system optimizations and freely update the visualization design.

\section{ACKNOWLEDGMENTS}

This work was supported by NSF grants 1527765, 156404, 1845638, and Google, Amazon, and Adobe Research awards.

\section{REFERENCES}

[1] Agrawal, S., Bruno, N., Chaudhuri, S., and Narasayya, V. R. Autoadmin: Self-tuning database systemstechnology. IEEE Data Eng. Bull. 29, 3 (2006), 7-15.

[2] Botello, C. Adobe InDesign CS6 Revealed. Cengage Learning, 2012.

[3] Bruno, N., ANd Chaudhuri, S. Constrained physical design tuning. Proceedings of the VLDB Endowment 1, 1 (2008), 4-15.

[4] Chaudhuri, S., and Narasayya, V. R. An efficient, cost-driven index selection tool for microsoft sql server. In $V L D B$ (1997), vol. 97, Citeseer, pp. $146-155$.

[5] Chen, Y., AND Wu, E. Monte carlo tree search for generating interactive data analysis interfaces. AAAI Workshop on Intelligent Process Automation (2020).

[6] Design, F. Figma: the collaborative interface design tool.(2017). Retrieved September 17 (2017), 2017.

[7] Hanrahan, P. Vizql: a language for query, analysis and visualization. In Proceedings of the 2006 ACM SIGMOD international conference on Management of data (2006), pp. 721-721.

[8] Moritz, D., Howe, B., AND Heer, J. Falcon: Balancing interactive latency and resolution sensitivity for scalable linked visualizations. In Proceedings of the 2019 CHI Conference on Human Factors in Computing Systems (2019), ACM, p. 694.

[9] Satyanarayan, A., Moritz, D., Wongsuphasawat, K., and Heer, J. Vega-lite: A grammar of interactive graphics. IEEE transactions on visualization and computer graphics 23, 1 (2016), 341-350.

[10] Tao, W., Liu, X., Wang, Y., Battle, L., Demiralp, Ç., Chang, R., and Stonebraker, M. Kyrix: Interactive pan/zoom visualizations at scale. In Computer Graphics Forum (2019), vol. 38, Wiley Online Library, pp. 529-540.

[11] Walenz, B., Gao, J., Sonmez, E., Tian, Y., Wen, Y., Xu, C., Adair, B., AND YANG, J. Fact checking congressional voting claims.

[12] Zhang, Q., Zhang, H., AND Wu, E. Mining precision interfaces from query logs. arXiv preprint arXiv:1904.02344 (2019). 